\title{
On Discrete-time Pursuit-evasion Games with Sensing Limitations
}

\author{
Shaunak D. Bopardikar (Corresponding author) \\ Department of Mechanical Engineering, \\ University of California, Santa Barbara, CA 93106 \\ Email: bshaunak@umail.ucsb.edu \\ Tel: $+1-805-893-2801$ \\ Francesco Bullo \\ Department of Mechanical Engineering, \\ University of California, Santa Barbara, CA 93106 \\ Email: bullo@engineering.ucsb.edu \\ João P. Hespanha \\ Department of Electrical Engineering, \\ University of California, Santa Barbara, CA 93106 \\ Email: hespanha@ece.ucsb.edu
}

FINAL MANUSCRIPT

Log Number: K07-494

\begin{abstract}
We address discrete-time pursuit-evasion games in the plane where every player has identical sensing and motion ranges restricted to closed discs of given sensing and stepping radii. A single evader is initially located inside a bounded subset of the environment and does not move until detected. We propose a Sweep-Pursuit-Capture pursuer strategy to capture the evader and apply it to two variants of the game: the first involves a single pursuer and an evader in a bounded convex environment and the second involves multiple pursuers and an evader in a boundaryless environment. In the first game, we give a sufficient condition on the ratio of sensing to stepping radius of the players that guarantees capture. In the second, we determine the minimum probability of capture, which is a function of a novel pursuer formation and independent of the initial evader location. The Sweep and Pursuit phases reduce both games to previously-studied problems with unlimited range sensing, and capture is achieved using available strategies. We obtain novel upper bounds on the capture time and present simulation studies that address the performance of the strategies under sensing errors, different ratios of sensing to stepping radius, greater evader speed and different number of pursuers.
\end{abstract}

\section{Index Terms}

Pursuit-evasion games, sensing limitations, cooperative control.

\section{Paper type: Regular paper}

Cover Note: Shaunak D. Bopardikar, Francesco Bullo and João P. Hespanha are with the Center for Control, Dynamical systems and Computation (CCDC), University of California at Santa Barbara, Santa Barbara, CA 93106, USA.

This material is based upon work supported in part by ARO MURI Award W911NF-05-1-0219, ONR Award N00014-071-0721 and by the Institute for Collaborative Biotechnologies through the grant DAAD19-03-D-0004 from the U.S. Army Research Office. Early versions this work appeared in the IEEE American Control Conference, in New York City, USA, 2007, titled "Sensing limitations in the Lion and Man problem" and "Cooperative pursuit with sensing limitations". 


\section{On Discrete-time Pursuit-evasion Games with Sensing Limitations}

\begin{abstract}
We address discrete-time pursuit-evasion games in the plane where every player has identical sensing and motion ranges restricted to closed discs of given sensing and stepping radii. A single evader is initially located inside a bounded subset of the environment and does not move until detected. We propose a Sweep-Pursuit-Capture pursuer strategy to capture the evader and apply it to two variants of the game: the first involves a single pursuer and an evader in a bounded convex environment and the second involves multiple pursuers and an evader in a boundaryless environment. In the first game, we give a sufficient condition on the ratio of sensing to stepping radius of the players that guarantees capture. In the second, we determine the minimum probability of capture, which is a function of a novel pursuer formation and independent of the initial evader location. The Sweep and Pursuit phases reduce both games to previouslystudied problems with unlimited range sensing, and capture is achieved using available strategies. We obtain novel upper bounds on the capture time and present simulation studies that address the performance of the strategies under sensing errors, different ratios of sensing to stepping radius, greater evader speed and different number of pursuers.
\end{abstract}

Index Terms-Pursuit-evasion games, sensing limitations, cooperative control.

\section{INTRODUCTION}

The game of pursuit can be posed as to determine a strategy for a pursuer (a team of pursuers) to capture an evader in a given environment. By capture, we mean that the evader and the pursuer (some pursuer) meet at the same location after a finite time. The aim of the pursuer (pursuers) is to capture the evader for any evader trajectory. The evader wins the game if it can avoid capture indefinitely. All the players have identical motion capabilities. Capture strategies are important in surveillance where we would like to detect and capture equally agile intruders. Another application is search-and-rescue operations where a worst-case capture strategy guarantees a rescue, in spite of any unpredictable motion of the victim.

The continuous time version of this game has been studied in [1], [2] and [3] to cite a few. Recently, the discrete-time version of the game has received significant attention. [4] describes sufficient conditions and a strategy for a single pursuer to capture an evader in a semi-open environment. This strategy has been extended in [5] to the case of multiple pursuers in an unbounded environment, to capture a single evader which is inside their convex hull. [6] and [7] describe pursuer strategies of moving towards, and towards the previous positions of the evader respectively, so that the distance to the evader is reduced to a finite, non-zero amount in finite time. The game has also been studied in different types of bounded environments, e.g., circular environment [6], curved environments [8]. Visibility-based pursuit evasion has been studied in a continuous-time setting in [9] and in polygonal environments in a discrete-time setting in [10].
In the context of sensing limitations, in continuous time formulations, [11] deals with a version of visibility limited to an angle, instead of the entire region. [12] considers a successive pursuit of multiple evaders by a single faster pursuer in the plane with sensing range limited to a finite disc. [13] proposes a multi-phase pursuit strategy for groups of pursuers with limited range sensing and has demonstrated its capture properties in bounded environments via simulation without formal proof. In discrete time formulations, [14] considers the problem on a graph, with the visibility of the pursuer limited to nodes adjacent to the current node of a pursuer. A framework which uses probabilistic models for sensing devices for the agents is described in [15] and [16].

We address the case of limited range sensing capability: a pursuer and an evader can sense each other only if the distance between them is less than or equal to a given sensing radius. We consider the discrete-time version with one or many pursuers and a single evader in a planar environment. The motion of each player is constrained to a stepping disc around it. The evader is initially located inside a bounded subset of the environment, which we term as the field. The players can leave the field but not the environment. The evader follows a reactive rabbit model, i.e., does not move until it senses a pursuer [14]. We present an algorithmic approach in the form of a Sweep-Pursuit-Capture strategy for the pursuer to capture the evader. We demonstrate this strategy using two variants of the pursuit-evasion game: the first involves a single pursuer and the evader in a bounded convex environment while the second considers multiple cooperating pursuers to capture the evader in a boundaryless environment.

In the first game, the pursuer sweeps the environment in a definite path until the evader is sensed, which must necessarily happen in finite time. This is analogous to the spanning-tree based coverage presented in [17]. We then establish how a GREEDY strategy of moving towards the last-sensed location of the evader, eventually reduces the present problem to a previously-studied one with unlimited sensing. The convexity assumption on the environment is required because otherwise, due to the limited sensing range, there exist environments similar to those considered in [10] and an evader strategy, such that the evader does not get detected again. Finally, we show how capture is achieved using the established LION strategy [4]. Our contributions are as follows: First, we present an analysis which provides a novel upper bound on the time required for the pursuit phase to terminate. This bound is an improvement compared to our earlier work [18]. Second, we obtain a sufficient condition on the ratio of sensing to stepping radius of the players for capture to take place in a given environment. Finally, we show that this condition is tight in the sense that if it is violated, then there exist sufficiently large 
environments, an evader strategy and initial positions for the players, that lead to evasion against the GREEDY strategy.

The second game is played with at least five cooperative pursuers in a boundaryless environment and the field is a bounded region known to the pursuers. Our contributions are as follows: First, we design a novel pursuer formation and a randomized SwEEP strategy for the pursuers to search the field. They succeed when they detect the evader inside a special capture region, which we characterize for the pursuer formation. We show that using our SWEEP strategy, the pursuers succeed with a certain probability which is a function of the pursuer formation and independent of the initial evader location. Next, we propose a cooperative pursuit strategy for the pursuers to confine the evader within their sensing discs. We show that using this pursuit strategy, the present problem is reduced to a previously-studied one with unlimited sensing. Finally, we show how capture is achieved using the established PLANES strategy [5]. We obtain novel upper bounds on the time for each phase in our strategy, which is an improvement compared to our earlier work [19]. Also, we present a simulation-based study of the performance of the strategies under sensing errors, different ratios of sensing to stepping radius, greater evader speed and different number of pursuers.

The inspiration for the cooperative strategy proposed in this paper has been derived from aspects of animal behavior. It is well known that predators hunt as a conjoined group, when it is less efficient to hunt alone. This behavior is also observed when the prey is large or can move as fast as the predators [20]. Further, predators show an inclination towards specialized behavior by maintaining a fixed formation during search and capture of preys [21]. Such specializations suggest that there may be configurations that are preferred during group hunting. Also, in the presence of sensing limitations, groups tend to maintain spacing between each other that is regulated by their sensory capabilities [22]. These facts give us additional hints towards designing capture-conducive predator formations. In this context, our analysis sheds light on how the maximum group size of the predators varies with prey availability and with the prey's nutrition value.

The paper is organized as follows: the problem's mathematical model and assumptions are presented in Section II. The individual phases of the sweep-pursuit-capture strategies and the corresponding main results for both the problems are presented in Section III. Due to space constraints, the proofs of all the results are presented in the online technical report [23]. Simulation results are presented in Section IV. Finally, in Section V, we study the relationship between pursuer group sizes and evader availability and its nutrition value.

\section{Problem Set-UP}

We assume a discrete-time model with alternate motion of the evader and the pursuers: the evader moving first. We assume that the players can sense each other precisely only if the distance between them is less than or equal to the sensing radius $r_{\text {sens }}$. Further, we assume that at each time instant, the players take measurements of each other before and after the evader's move, as shown in Figure 1. Define $\mathcal{Q}_{\phi}:=\mathcal{Q} \cup \phi$, where $\mathcal{Q} \subseteq \mathbb{R}^{2}$ denotes the environment and $\phi$ is the null element. The null element will be used to denote a lack of measurement in our limited range sensing model. Let $\mathcal{G} \subset \mathcal{Q}$ denote the field, i.e., the region that initially contains the evader. The evader follows a reactive rabbit model - moves only after being detected for the first time. We assume that the pursuers know the field $\mathcal{G}$ and the environment $\mathcal{Q}$. The goal of the pursuer(s) is to capture the evader, i.e., a pursuer and the evader are at the same position at some finite time. Evasion is said to occur if the pursuer cannot capture the evader. We describe the Sweep-Pursuit-Capture strategy for the following problems:

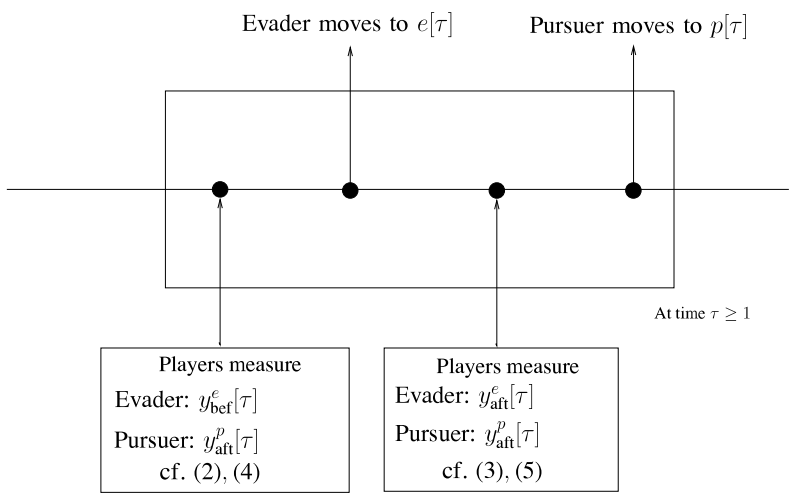

Fig. 1. A snapshot of each time instant $\tau \in\{1,2, \ldots\}$ in our alternate motion model. Players take measurements before and after the evader's move.

\section{A. Single pursuer problem}

We have a bounded convex environment $\mathcal{Q} \subset \mathbb{R}^{2}$ and the field $\mathcal{G}=\mathcal{Q}$. Let $e[t]$ and $p[t]$ denote the absolute positions of the evader and the pursuer respectively, at time $t \in \mathbb{Z}_{\geq 0}$. The discrete-time equations of motion are

$$
\begin{aligned}
& e[t]=e[t-1]+u^{e}\left(e[t-1],\left\{y_{\mathrm{bef}}^{e}[\tau]\right\}_{\tau=1}^{t},\left\{y_{\mathrm{aft}}^{e}[\tau]\right\}_{\tau=1}^{t-1}\right), \\
& p[t]=p[t-1]+u^{p}\left(p[t-1],\left\{y_{\mathrm{bef}}^{p}[\tau]\right\}_{\tau=1}^{t},\left\{y_{\mathrm{aft}}^{p}[\tau]\right\}_{\tau=1}^{t}\right),
\end{aligned}
$$

where at the $\tau^{t h}$ time instant, $y_{\text {bef }}^{e}[\tau], y_{\text {aft }}^{e}[\tau] \in \mathcal{Q}_{\phi}$ are the measurements of the pursuer's position taken by the evader before and after the evader's move, as shown in Figure 1. The parentheses notation $\left\{y_{\text {bef }}^{p}[\tau]\right\}_{\tau=1}^{t}$ denotes the set $\left\{y_{\text {bef }}^{p}[1], y_{\text {bef }}^{p}[2], \ldots, y_{\text {bef }}^{p}[t]\right\}$. Due to limited range sensing model, for $\tau \in\{1, \ldots, t\}$, we define

$$
y_{\text {bef }}^{e}[\tau]= \begin{cases}p[\tau-1], & \text { if }\|p[\tau-1]-e[\tau-1]\| \leq r_{\text {sens }}, \\ \phi, & \text { otherwise. }\end{cases}
$$

For notational convenience, we define $\left\{y_{\text {aft }}^{p}[\tau]\right\}_{\tau=1}^{t-1}=\phi$ for the initial time $t=1$. For $t \geq 2$ and for $\tau \in\{1, \ldots, t-1\}$, we have

$$
y_{\mathrm{aft}}^{e}[\tau]= \begin{cases}p[\tau-1], & \text { if }\|p[\tau-1]-e[\tau]\| \leq r_{\mathrm{sens}} \\ \phi, & \text { otherwise. }\end{cases}
$$

Similarly, at the $\tau^{t h}$ time instant, $y_{\text {bef }}^{p}[\tau], y_{\text {aft }}^{p}[\tau] \in \mathcal{Q}_{\phi}$ are the measurements of the evader's position taken by the 
pursuer before and after the evader's move respectively, as shown in Figure 1. Due to limited range sensing model, for $\tau \in\{1, \ldots, t\}$, we have

$$
y_{\mathrm{bef}}^{p}[\tau]= \begin{cases}e[\tau-1], & \text { if }\|e[\tau-1]-p[\tau-1]\| \leq r_{\mathrm{sens}}, \\ \phi, & \text { otherwise. }\end{cases}
$$

For $\tau \in\{1, \ldots, t\}$, we have

$$
y_{\mathrm{aft}}^{p}[\tau]= \begin{cases}e[\tau], & \text { if }\|e[\tau]-p[\tau-1]\| \leq r_{\mathrm{sens}}, \\ \phi, & \text { otherwise. }\end{cases}
$$

The functions $u^{e}: \mathcal{Q} \times \underbrace{\mathcal{Q}_{\phi} \times \cdots \times \mathcal{Q}_{\phi}}_{2 t-1 \text { times }} \rightarrow \mathcal{Q}$ and $u^{p}:$ $\mathcal{Q} \times \underbrace{\mathcal{Q}_{\phi} \times \cdots \times \mathcal{Q}_{\phi}}_{2 t \text { times }} \rightarrow \mathcal{Q}$ are termed as strategies for the evader and pursuer respectively. The apparent lack of symmetry between the number of arguments in the strategies of the evader and the pursuer is due to the alternate motion model. We assume that both players can move with a maximum step size of $r_{\text {step }}$, that is,

$$
\left\|u^{e}\right\| \leq r_{\text {step }}, \quad\left\|u^{p}\right\| \leq r_{\text {step }}
$$

The sensing radius, $r_{\text {sens }}$, is $\kappa$ times the motion radius, $r_{\text {step }}$. We assume $\kappa$ is greater than 1, i.e., both players can sense further than they can move. From the reactive rabbit model for the evader, we have $u^{e}=0$ until the evader is detected. After this happens, the single pursuer problem consists of determining $u^{p}$ that guarantees capture for any evader strategy, $u^{e}$. This problem is described by two key parameters: the ratio of sensing to stepping radius $\kappa$ and the ratio of the diameter of the environment to the stepping radius $\frac{\operatorname{diam}(\mathcal{Q})}{r_{\text {step }}}$.

\section{B. Multiple pursuer problem}

We have a total of $N \geq 5$ pursuers that can communicate among themselves the location of a sensed evader as well as their own position with respect to a fixed, global reference frame. The environment $\mathcal{Q}$ is $\mathbb{R}^{2}$ and the field $\mathcal{G}$ is a bounded subset of $\mathbb{R}^{2}$. Define $\mathbb{R}_{\phi}^{2}:=\mathbb{R}^{2} \cup \phi$. Let $p_{j}[t]$ denote the absolute positions of the $j^{t h}$ pursuer at time $t$ for every $j \in$ $\{1, \ldots, N\}$. Analogous to (1), the discrete-time equations of motion are

$$
\begin{aligned}
& e[t]=e[t-1]+u^{e}\left(e[t-1],\left\{y_{\mathrm{bef}}^{e}[\tau]\right\}_{\tau=1}^{t},\left\{y_{\mathrm{aft}}^{e}[\tau]\right\}_{\tau=1}^{t-1}\right), \\
& p_{j}[t]=p_{j}[t-1] \\
& +u^{p_{j}}\left(\left\{\left\{p_{j}[\tau]\right\}_{j=1}^{N}\right\}_{\tau=1}^{t-1},\left\{y_{\mathrm{bef}}^{p}[\tau]\right\}_{\tau=1}^{t},\left\{y_{\mathrm{aft}}^{p}[\tau]\right\}_{\tau=1}^{t}\right),
\end{aligned}
$$

where at the $\tau^{t h}$ time instant, $y_{\text {bef }}^{e}[\tau], y_{\text {aft }}^{e}[\tau] \in \underbrace{\mathbb{R}_{\phi}^{2} \times \cdots \times \mathbb{R}_{\phi}^{2}}_{N \text { times }}$ denote the sets of measurements of the pursuers' positions taken by the evader before and after its move. Similarly, $y_{\text {bef }}^{p}[\tau], y_{\text {aft }}^{p}[\tau] \in \mathbb{R}_{\phi}^{2}$ are the measurements of the evader's position taken by the pursuers before and after the evader's move. The measurements are given by expressions analogous to (2)-(5). Akin to the single pursuer problem, the functions $u^{e}: \mathbb{R}^{2} \times \underbrace{\mathbb{R}_{\phi}^{2} \times \cdots \times \mathbb{R}_{\phi}^{2}}_{(2 t-1) N \text { times }} \rightarrow \mathbb{R}^{2}$ and $u^{p_{j}}: \underbrace{\mathbb{R}_{\phi}^{2} \times \cdots \times \mathbb{R}_{\phi}^{2}}_{(t-1) N \text { times }} \times$ $\underbrace{\mathbb{R}_{\phi}^{2} \times \cdots \times \mathbb{R}_{\phi}^{2}}_{2 t \text { times }} \rightarrow \mathbb{R}^{2}$ for every $j \in\{1, \ldots, N\}$, are strategies for the evader and pursuers respectively. The constraint on the maximum step size, given by (6), holds for the evader and every pursuer. Due to the reactive rabbit model for the evader, $u^{e}=0$ until it is detected by the pursuers for the first time.

The multiple pursuer problem consists of designing a pursuer formation and a corresponding strategy that guarantees capture of the evader. This problem is described by the following key parameters: the ratio of sensing to stepping radius of the players $\kappa$, the ratio of the diameter of the field to the stepping radius $\frac{\operatorname{diam}(\mathcal{G})}{r_{\text {step }}}$, and the number of pursuers $N$.

\section{The Sweep-Pursuit-Capture Strategies And MAIN RESULTS}

In this section, we describe the Sweep-Pursuit-Capture strategies for both problems and the corresponding main results.

We first introduce the following weak notion of capture.

Definition III.1 (Trap) The evader is trapped within the sensing radius (resp. radii) of the pursuer (resp. pursuers) if for any evader strategy $u^{e}$, the motion disc of the evader is completely contained in the sensing disc of the pursuer (resp. union of the sensing discs of the pursuers) after a finite time.

To be specific, the evader is trapped at time instant $T_{\text {trap }}$ if for any evader strategy,

$$
y_{\text {bef }}^{p}\left[T_{\text {trap }}\right]=e\left[T_{\text {trap }}-1\right], \quad \text { and } \quad y_{\text {aft }}^{p}\left[T_{\text {trap }}\right]=e\left[T_{\text {trap }}\right] .
$$

The idea behind Sweep-Pursuit-Capture strategies is to detect the evader and pursue it so as to trap it. Next, we show that the evader remains trapped for all subsequent time instants and that capture is achieved by using strategies that were developed for the unlimited range sensing version of the respective game. This principle applies to both versions of the problem.

\section{A. Single pursuer problem}

We first present each phase of the strategy for the single pursuer problem.

1) Sweep phase - SwEEP strategy: Let $\operatorname{diam}(\mathcal{Q})$ denote the diameter of $\mathcal{Q}$. The SWEEP strategy is to move with maximum step size along a path, as shown in Figure 2 such that the union of the sensing discs of the pursuer at the end of each step until the end of this phase contains $\mathcal{Q}$. We term such a path a sweeping path for $\mathcal{Q}$. Let $t_{\text {sweep }}$ denote the time taken for this strategy to terminate. We have the following result.

Lemma III.2 (SWEEP strategy) In the single pursuer problem with parameters $\kappa$ and $\frac{\operatorname{diam}(\mathcal{Q})}{r_{\text {step }}}$, the time $t_{\text {sweep }}$ taken by the SWEEP is at most $\left\lceil\frac{\operatorname{diam}(\mathcal{Q})}{2 \kappa r_{\text {step }}}\right\rceil\left(\left\lceil\frac{\operatorname{diam}(\mathcal{Q})}{r_{\text {step }}}\right\rceil+\lceil\kappa\rceil\right)$ steps. 


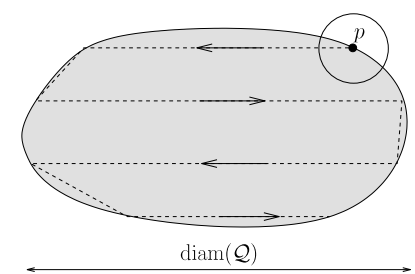

Fig. 2. A sweeping path to detect the evader in the Single pursuer problem using the SWEEP strategy.

2) Pursuit phase - GREEDY strategy: Once the evader is detected, the GREEDY strategy for the pursuer is to move towards the last sensed position of the evader with maximum step size. This strategy has the property that the pursuer senses the evader's position at every successive time instant. Let $t_{\text {trap }}$ denote the trapping time, i.e., the time taken by the pursuer to trap the evader after detecting it. We now present our main result for the GREEDY strategy.

Theorem III.3 (GREEDY strategy) In the single pursuer problem with parameters $\kappa$ and $\frac{\operatorname{diam}(\mathcal{Q})}{r_{\mathrm{step}}}$, if $\kappa>\sqrt{2+2 \cos \beta_{\mathrm{c}}}$, where

$$
\beta_{\mathrm{c}}:=\frac{\sqrt{3}}{4 \kappa}\left\lceil\frac{\operatorname{diam}(\mathcal{Q})}{2 \kappa r_{\text {step }}}\right\rceil^{-1} \arctan \frac{1}{8 \kappa},
$$

then the GREEDY strategy has the following properties:

(i) the pursuer traps the evader within its sensing radius, and

(ii) the trapping time $t_{\text {trap }}$ satisfies

$t_{\text {trap }} \leq\left(\left\lceil\frac{\log \left(\frac{\sqrt{\kappa^{2}-\sin ^{2} \beta_{\mathrm{c}}}-\cos \beta_{\mathrm{c}}-1}{\kappa-1}\right)}{\log \left(1-\frac{1-\cos \beta_{\mathrm{c}}}{\kappa}\right)}\right\rceil+1\right)\left\lceil\frac{\operatorname{diam}(\mathcal{Q})}{2 \kappa r_{\text {step }}}\right\rceil$.

Furthermore, if $\kappa>2$, then as $\left(\operatorname{diam}(\mathcal{Q}) / r_{\text {step }}\right) \rightarrow+\infty$, $t_{\text {trap }} \in O\left(\left(\operatorname{diam}(\mathcal{Q}) / r_{\text {step }}\right)^{3}\right)$.

Theorem III.3 is tight in the sense that if the condition on $\kappa$ is violated then there exist sufficiently large environments, an evader strategy and initial positions for the players, that lead to evasion against the GREEDY pursuer strategy. This is described by the following result.

Proposition III.4 (Evasion) Given a single pursuer problem with parameters $\kappa$ and $\frac{\operatorname{diam}(\mathcal{Q})}{r_{\text {step }}}$ such that $\kappa \leq \sqrt{2+2 \cos \beta_{\mathrm{c}}}$, where $\beta_{\mathrm{c}}$ is given by (8), and $\mathcal{Q}$ contains a circle of radius $\frac{r_{\text {step }}}{\sqrt{4-\kappa^{2}}}$, then there exist an evasion strategy and initial positions of the players for which the pursuer's GREEDY strategy fails to trap the evader.

Figure 3 illustrates this evasion strategy under the conditions required by Proposition III.4.

3) Capture phase - LION strategy: Once the evader is trapped within the sensing range of the pursuer, the pursuer employs the LION strategy from [4] to complete the capture. For the sake of completeness, we now give a brief description of the LION strategy, adapted to the present problem setting.

The LION strategy can be applied to this phase as follows:

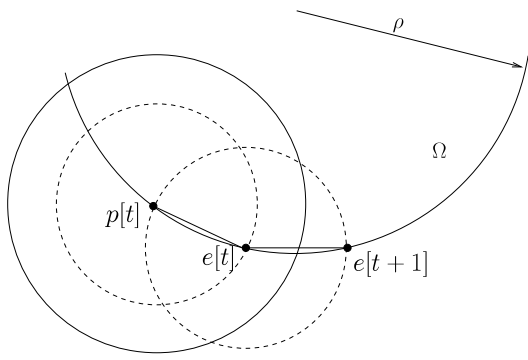

Fig. 3. Illustrating evasion. The dotted circles are the player's motion discs and the solid circle is the pursuer's sensing disc. $e[t]$ and $p[t]$ are on the circle $\Omega$ described in Proposition III.4 such that $\|e[t]-p[t]\|=r_{\text {step. }}$ Evader chooses to move to $e[t+1]$ on $\Omega$ with full step size.

(i) Prior to its $(t+1)^{t h}$ move, the pursuer constructs the line $e[t] p[t]$, as shown in Figure 4. Let this line intersect the boundary of the environment at a point $X[t]$ such that $p[t]$ lies between $e[t]$ and $X[t]$.

(ii) The pursuer then also constructs the line $e[t+1] X[t]$ and moves to the intersection of this line with the circle centered at $p[t]$ and of radius $r_{\text {step }}$. Of the two possible intersection points, the pursuer selects the one closer to $e[t+1]$.

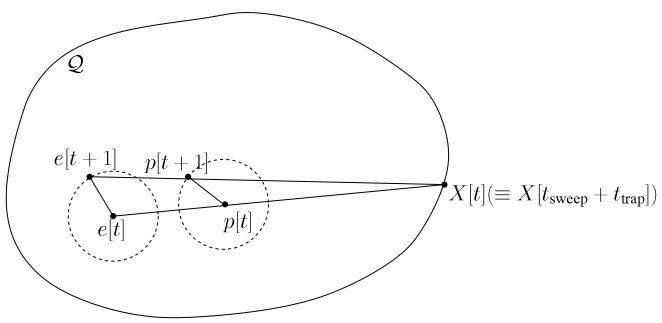

Fig. 4. Single pursuer problem: Using the LION strategy to capture the evader. The dotted circles represent the motion discs of the players.

This construction guarantees that the point $X[t]$ remains the same as the point $X\left[t_{\text {sweep }}+t_{\text {trap }}\right]$, for every $t \geq t_{\text {sweep }}+t_{\text {trap }}$, where $t_{\text {sweep }}+t_{\text {trap }}$ is the time at the end of the pursuit phase. Let $t_{\text {cap }}$ be the time taken by the pursuer to capture the evader after trapping it. We have the following result.

Theorem III.5 (LION strategy [4]) In the single pursuer problem with parameters $\kappa$ and $\frac{\operatorname{diam}(\mathcal{Q})}{r_{\text {step }}}$, after trapping the evader within the sensing radius and using the Lion strategy,

(i) the distance, $\|p[t]-e[t]\|$, is a non-increasing function of time,

(ii) the pursuer captures the evader,

(iii) $t_{\text {cap }}$ is at most $\left[\left(\frac{\operatorname{diam}(\mathcal{Q})}{r_{\text {step }}}\right)^{2}\right]$ steps.

Thus, our problem with limited sensing is solved because once the evader is trapped within the pursuer's sensing radius, it remains trapped until capture, from part (i) of Theorem III.5. We have also obtained an upper bound on the total time to capture, i.e., $t_{\text {sweep }}+t_{\text {trap }}+t_{\text {cap }}$.

\section{B. Multiple pursuer problem}

This section describes the sweep-pursuit-capture strategy for multiple pursuers and the corresponding results. We assume that $\kappa \geq 4$ and $N \geq 5$. We define the following formation. 
Definition III.6 (Trapping chain) $A$ group of $N \geq 5$ pursuers $\left\{p_{1}, \ldots, p_{N}\right\}$ are said to be in a trapping chain formation if

(i) $p_{2}, \ldots, p_{N-1}$ are placed counterclockwise on a semicircle with diameter equal to $\left\|p_{2}-p_{N-1}\right\|$,

(ii) for all $j \in\{1, \ldots, N-1\}$

$$
\left\|p_{j}-p_{j+1}\right\|=r_{\text {step }} \sqrt{4 \kappa^{2}-25} \text {, and }
$$

(iii) $p_{1}, p_{2}, p_{N-1}, p_{N}$ are on the vertices of a rectangle such that the polygon with vertices $\left\{p_{1}, \ldots, p_{N}\right\}$, in that order, is convex (cf. Figure 5).

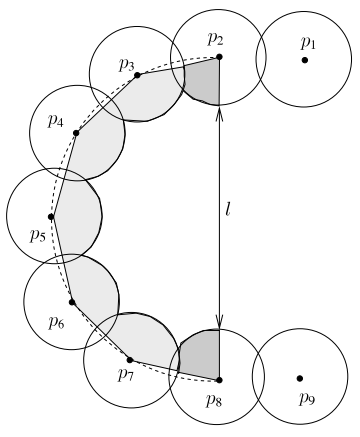

Fig. 5. A trapping chain formation for $N=9$ pursuers. The circles around the pursuers denote their sensing ranges. The lightly shaded region denotes the capture region and the darkly shaded region along with the lightly shaded one denotes the extended capture region.

We now describe the Sweep-Pursuit-Capture strategy for the multiple pursuer problem.

1) Sweep phase - SWEEP strategy: The pursuers begin by placing themselves in a trapping chain formation. We define the capture region $\mathcal{S}$ for a trapping chain by

$$
\mathcal{S}=\bigcup_{j \in\{3, \ldots, N-2\}} \mathcal{B}_{p_{j}}\left(r_{\text {sens }}\right) \cap \mathrm{Co}\left\{p_{2}, \ldots, p_{N-1}\right\},
$$

where $\mathcal{B}_{p_{j}}\left(r_{\text {sens }}\right) \subset \mathbb{R}^{2}$ denotes the sensing disc of pursuer $p_{j}$ and $\operatorname{Co}\left\{p_{2}, \ldots, p_{N-1}\right\} \subset \mathbb{R}^{2}$ denotes the interior of the convex hull of $\left\{p_{2}, \ldots, p_{N-1}\right\}$. The lightly shaded region in Figure 5 is the proposed capture region, $\mathcal{S}$, for the trapping chain. In the sweep phase, pursuers wish to detect the evader within the capture region. We consider a square region of length equal to diameter of the region $\mathcal{G}, \operatorname{diam}(\mathcal{G})$ that contains the field $\mathcal{G}$. The pursuers sweep this square region in the direction of the normal to $p_{1} p_{N}$, outward with respect to the convex hull of the pursuers. For a trapping chain shown in Figure 5, we define the effective length $l$ as

$$
l:=\left\|p_{1}-p_{N}\right\|-2 r_{\text {sens }}=r_{\text {step }}\left(\frac{\sqrt{4 \kappa^{2}-25}}{\sin \left(\frac{\pi}{2(N-3)}\right)}-2 \kappa\right) .
$$

As the pursuers move in the direction described earlier, they clear a rectangular strip of length $\operatorname{diam}(\mathcal{G})$ and width $l+4 r_{\text {sens }}$. The SwEEP strategy for the pursuers is as follows.

(i) Choose the first rectangular strip at a random distance $l_{0}$ from one edge of the square region containing $\mathcal{G}$ and sweep it length-wise. The distance $l_{0}$ is a uniform random variable taking values in the interval $\left[-2 r_{\text {sens }}, l+2 r_{\text {sens }}\right]$. Here, negative $l_{0}$ implies that some of the pursuers may begin sweeping from outside $\mathcal{G}$.

(ii) Form a sweeping path for the square region and sweep along adjacent strips as shown in Figure 6.

The shaded region in Figure 6 refers to the area that would fall in the proposed capture region $\mathcal{S}$. Now we are interested in determining the probability that an evader falls in the shaded region in Figure 6. That is given by the following result.

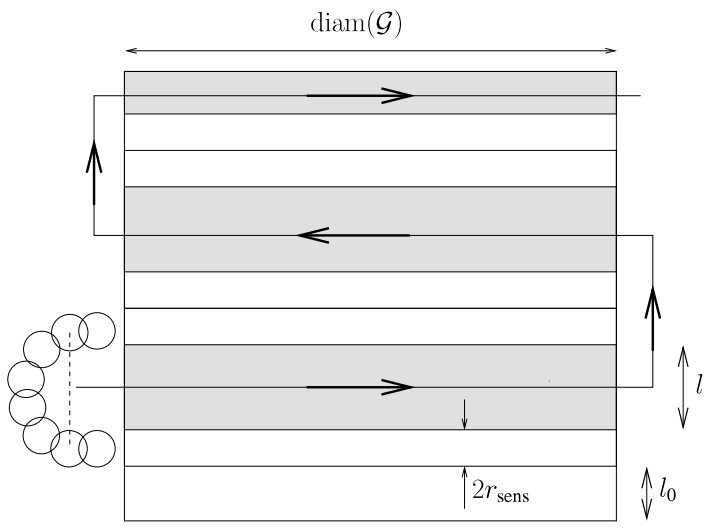

Fig. 6. Multiple pursuer problem: SweEP strategy. The shaded region represents the region swept by the capture region of the trapping chain.

Theorem III.7 (SWEEP strategy) In the multiple pursuer problem with parameters $\kappa, \frac{\operatorname{diam}(\mathcal{G})}{r_{\text {step }}}$ and $N$, for any probability distribution for the initial position of the evader with support on $\mathcal{G}$, using the SWEEP strategy,

(i) the probability $P$ of detecting the evader inside $\mathcal{S}$ for a group of pursuers in a trapping chain, satisfies

$$
P \geq \frac{l}{l+4 r_{\text {sens }}} \geq 1-\frac{2 \pi \kappa}{\left(\sqrt{4 \kappa^{2}-25}(N-3)+2 \pi \kappa\right)} \text {, and }
$$

(ii) the time $t_{\text {sweep }}$ taken by the SWEEP strategy to terminate satisfies

$$
\begin{aligned}
t_{\text {sweep }} \leq\left\lceil\frac{\operatorname{diam}(\mathcal{G})}{r_{\text {step }}}(\right. & \left.\left.\frac{\pi / 2}{\sqrt{4 \kappa^{2}-25}(N-3)+\pi \kappa}\right)\right\rceil \\
& \times\left\lceil\frac{\operatorname{diam}(\mathcal{G})}{r_{\text {step }}}+2 \sqrt{4 \kappa^{2}-25} N\right\rceil .
\end{aligned}
$$

Remark III.8 The minimum probability $P$ of the pursuers detecting the evader inside the capture region by using the SwEEP strategy is independent of the evader's location. Hence, the best that the evader can do in the present framework is to locate itself initially with a uniform probability in $\mathcal{G}$.

We shall see that the pursuers win when the evader is detected in $\mathcal{S}$ by the pursuers. Otherwise, there exists a path for the evader such that it can avoid being captured indefinitely.

2) Pursuit phase - Circumcenter strategy: If the evader is detected within the proposed capture region at time $t_{\text {sweep }}$, the pursuers need to ensure that they trap the evader within their sensing ranges. Before we describe the strategy for the pursuit phase, consider the following possibility: if the evader steps into the darkly shaded region of the sensing range of 
$p_{2}$ (or of $\left.p_{N-1}\right)$, then $p_{2}$ (resp. $p_{N-1}$ ) can use the GREEDY strategy (ref. Section III-A2). By moving towards the evader, the evader's motion disc gets contained inside that pursuer's sensing disc and thus the evader gets trapped. This motivates us to define an extended capture region $\mathcal{S}^{e}$ for the trapping chain by

$$
\mathcal{S}^{e}=\bigcup_{j \in\{2, \ldots, N-1\}} \mathcal{B}_{p_{j}}\left(r_{\text {sens }}\right) \cap \operatorname{Co}\left\{p_{2}, \ldots, p_{N-1}\right\} .
$$

The darkly shaded region along with the lightly shaded region in Figure 5 is the extended capture region $\mathcal{S}^{e}$ for the trapping chain.

We now present the following pursuit strategy. At each time step $t \geq t_{\text {sweep }}$

(i) While $e[t+1] \notin \mathcal{S}^{e}[t]$, the pursuers $p_{2}, \ldots, p_{N-1}$ move towards the circumcenter ${ }^{1} O$ of the triangle formed by $p_{2}\left[t_{\text {sweep }}\right], e\left[t_{\text {sweep }}\right]$ and $p_{N-1}\left[t_{\text {sweep }}\right]$ with maximum step. Pursuers $p_{1}$ and $p_{N}$ move parallel to $p_{2}$ and $p_{N-1}$ respectively.

(ii) Otherwise, one of the pursuers which senses the evader directly, makes a GREEDY move (ref. Section III-A2) towards the evader and the others move parallel to that pursuer with the maximum step.

One such move is shown in Figure 7. In case (i) of the strategy, note that the pursuers may not sense the evader in every subsequent move. But they will encircle the evader by closing the trapping chain around it and then shrink the enclosed region around the evader. We have the following result.

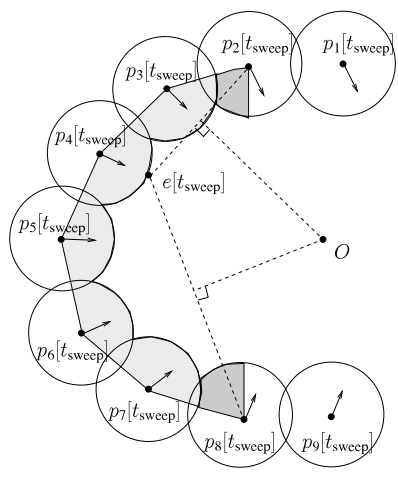

Fig. 7. Multiple pursuer problem: CiRCUMCENTER strategy. At time $t_{\text {sweep }}$ the evader position is sensed by $p_{4}$. Pursuers $p_{2}, \ldots, p_{8}$ move towards $\mathrm{O}$, the circumcenter of triangle formed by $p_{2}, e$ and $p_{8} . p_{1}$ and $p_{9}$ move parallel to $p_{2}$ and $p_{8}$ respectively. The circles around the pursuers represent their sensing discs.

Theorem III.9 (CIRCUMCENTER strategy) In the multiple pursuer problem with parameters $\kappa$, $\frac{\operatorname{diam}(\mathcal{G})}{r_{\text {step }}}$ and $N$, starting from a trapping chain formation, if the evader is detected with $e\left[t_{\text {sweep }}\right] \in \mathcal{S}\left[t_{\text {sweep }}\right]$, then using the CIRCUMCENTER strategy,

(i) the pursuers trap the evader within their sensing radii,

(ii) the trapping time $t_{\text {trap }}$ satisfies

$$
t_{\text {trap }} \leq \sqrt{4 \kappa^{2}-25} N\left(1+\frac{1}{2 \sin \phi}\right)
$$

\footnotetext{
${ }^{1}$ The circumcenter of a triangle is the unique point in the plane which is equidistant from all of its three vertices.
}

where

$$
\phi(\kappa)=\frac{\pi}{4}-\arctan \left(\frac{\kappa}{\sqrt{3 \kappa^{2}-25}}\right), \text { and }
$$

(iii) at that time instant, the evader is inside the pursuers' convex hull in such a way that

$$
\mathcal{B}_{\frac{r_{\text {step }}}{2}}\left(e\left[t_{\text {sweep }}+t_{\text {trap }}\right]\right) \subset \operatorname{Co}\left\{p_{1}, \ldots, p_{N}\right\}\left[t_{\text {sweep }}+t_{\text {trap }}\right],
$$

where the notation $\mathcal{B}_{r}(a)$ refers to the closed disc of radius $r$ centered at point $a$ in the plane.

The CIRCUMCENTER strategy guarantees trapping of the evader even without pursuers $p_{1}$ and $p_{N}$. But in that case, the inclusion in (11), which will be required to establish an upper bound on the time for the capture phase that follows, is not guaranteed.

3) The Capture phase - PLANES strategy: Once the evader is trapped within the sensing ranges of the pursuers, the pursuers use the PLANES strategy from [5] to capture the evader. Before stating our results, we reproduce this strategy for completeness.

Let the time at the end of the pursuit phase be $t_{\text {sweep }}+t_{\text {trap }}$ and the evader be inside the convex hull of the pursuers as in (11) (cf. Figure 8(a)). For $t \geq t_{\text {sweep }}+t_{\text {trap }}$ and for every pursuer $p_{j}$ :

- Draw the line $h_{j}[t+1]$ through $e[t+1]$, parallel to the line joining $e[t]$ and $p_{j}[t]$, as shown in Figure 8(b).

- Move to the point closest to $e[t+1]$ on the line $h_{j}[t+1]$ with maximum step size.

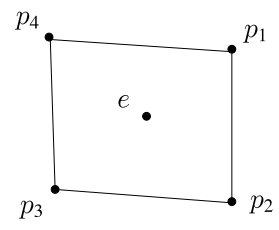

(a) Evader inside pursuers' convex hull

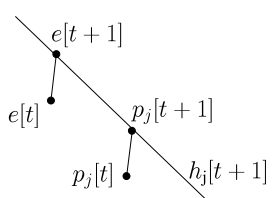

(b) PlANES strategy. Illustrating a pursuer move.
Fig. 8. Algorithm Planes

Theorem III.9 shows that use of the CIRCUMCENTER strategy in the pursuit phase leads to the evader being trapped and inside the convex hull of the pursuers. Now capture follows from the following theorem, which was partly inspired by the results on the PLANES strategy in [5].

Theorem III.10 (PLANES strategy) In the multiple pursuer problem with parameters $\kappa, \frac{\operatorname{diam}(\mathcal{G})}{r_{\text {ste }}}$ and $N$, let the evader be trapped inside the convex hull of the pursuers such that property (11) is satisfied. If every pursuer follows the PLANES strategy, then

(i) the distances, $\left\|p_{j}[t]-e[t]\right\|$ for every $j \in\{1, \ldots, N\}$, are non-increasing functions of time,

(ii) the pursuers capture the evader and

(iii) the time $t_{\text {cap }}$ taken in the capture phase is at most $18 \kappa \sqrt{4 \kappa^{2}-25} N$. 
Item (i) of Theorem III.10 implies that once the evader is trapped within the sensing ranges of the pursuers, it remains trapped within their sensing ranges at the end of every pursuer move. The capture is now complete and we obtained a novel upper bound on the total time to capture, i.e., $t_{\text {sweep }}+t_{\text {trap }}+t_{\text {cap }}$.

\section{Simulation Results}

We now present simulation studies to investigate the robustness of the algorithms to sensing errors. We study the performance of the algorithms in several cases such as different sensing to stepping radius ratio and faster evader. We also study the case of different number of pursuers in the multiple pursuer problem. All simulations were run using MATLAB ${ }^{\circledR}$.

We assume two types of sensing error models:

Gaussian errors: We assume zero-mean additive Gaussian measurement errors in the position of the evader with a standard deviation given by

$$
\sigma_{j}[t]=\epsilon\left\|p_{j}[t]-e[t]\right\|
$$

for some constant $\epsilon \geq 0$. This means that the uncertainty in the location of the evader increases with its distance from a pursuer. The evader is defined to be captured if the probability of the evader being inside the motion disc of the pursuer before the pursuer's move is more than a certain threshold. In other words, for some $t$ and for some pursuer $p_{j}$,

$$
\mathcal{B}_{\sigma_{j}[t]}\left(y_{\text {bef }}^{p}[t]\right) \subset \mathcal{B}_{r_{\text {step }}}\left(p_{j}[t-1]\right),
$$

where $\mathcal{B}_{\sigma_{j}[t]}\left(y_{\text {bef }}^{p}[t]\right)$ denotes the circular region of radius $\sigma_{j}[t]$ centered at $y_{\text {bef }}^{p}[t]$.

Non-Gaussian errors: The measured distance is given by $\left(1+\epsilon^{*}\right)\left\|p_{j}[t]-e[t]\right\|$, where $\epsilon^{*}$ is a random variable uniformly distributed in the interval $[-\epsilon, \epsilon]$, where $\epsilon \geq 0$ is the specified error parameter. With respect to angular measurements, if $\theta_{a}$ is the actual angular location of the evader with respect to a local reference frame of a pursuer, then the measured angular location is given by $\theta_{a}+\epsilon_{\theta}$, where $\epsilon_{\theta}$ is a random variable uniformly distributed in the interval $[-\Delta \theta, \Delta \theta]$, where the value of $\Delta \theta$ was chosen to be 1 degree. The evader is captured in this model if $y_{\text {bef }}^{p}[t] \in \mathcal{B}_{r_{\text {step }}}\left(p_{j}[t-1]\right)$.

\section{A. Single pursuer problem}

Under the considered noisy sensor models:

- The SWEEP strategy remains unchanged. It terminates when an evader measurement is available.

- For the GREEDY and LION strategies, the pursuer uses the noisy measurements of the evader position instead of the true position $e[t]$ to compute its next position.

For the evader's motion, we assume that it moves away from the pursuer with some randomization, while avoiding the boundary. Specifically,

- if the evader is not close to the boundary of the environment, then it chooses to move to a point on its motion circle, selected uniformly randomly in a sector with angle 0.2 radians. This sector is placed symmetrically along the line $e[t] p[t]$ and away from the pursuer.

- If the boundary is visible to the evader, then it chooses to move to a point $e[t+1]$ on its motion circle such that $\angle e[t+1] e[t] p[t]=\pi-0.2$. Of the two points possible, the evader chooses that point which is further away from the boundary. In other words, when the evader reaches the boundary, it chooses to move to a point that is away from the pursuer as well as not very close to the boundary.

For our simulations, the environment is a circle with diameter 40 units. We assume $\kappa=5$ units and $r_{\text {step }}=1$ unit. The initial position of the evader was chosen uniformly randomly in the environment. An upper limit of 2,000 time steps was set to decide whether the strategy terminated in a success.

The following is a summary of our findings:

(i) Performance of the strategy with noisy measurements: The plots of probability of success of the strategy and average capture times after detection (given that the strategy terminates with capture) for both noise models versus the respective error parameters $\epsilon$ are shown in Figure 9. We observe a similar trend in the performance of the strategy in both noise models.
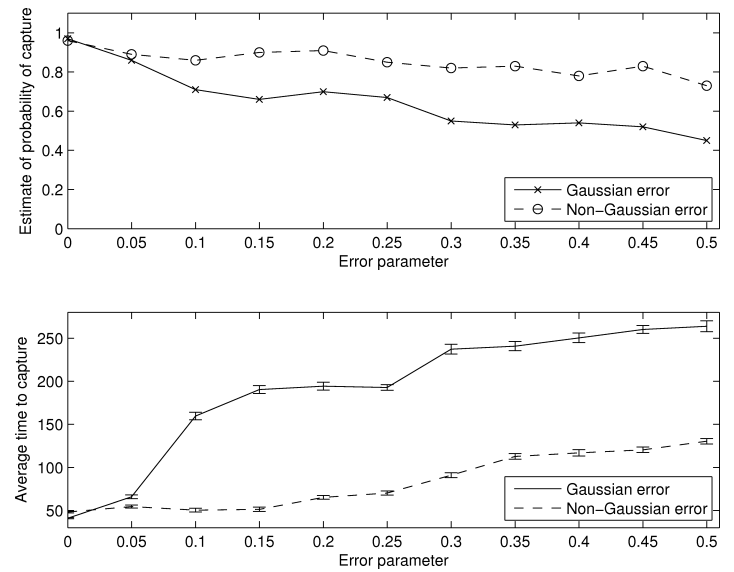

Fig. 9. Effect of measurement noise in the single pursuer problem. For a particular evader strategy, we study how the capture probability and average capture time given that the strategy succeeds, vary with the noise parameter $\epsilon$, under Gaussian and Non-gaussian error models. In the top figure, an interval of \pm 0.1 (not shown to preserve clarity) about the probability estimates is the $95 \%$ confidence interval given by $\left[P(\epsilon)-2 \sqrt{\frac{0.25}{n}}, P(\epsilon)+2 \sqrt{\frac{0.25}{n}}\right]$, where $n=100$ is the number of trials [24]. In the bottom figure, the vertical bars give a $95 \%$ confidence interval about the average capture time $T(\epsilon)$ which is given by $\left[T(\epsilon)-2 \sqrt{\frac{S D(\epsilon)}{n P(\epsilon)}}, T(\epsilon)+2 \sqrt{\frac{S D(\epsilon)}{n P(\epsilon)}}\right]$, where $S D(\epsilon)$ is the standard deviation in the capture time, $P(\epsilon)$ is the estimated probability of success and $n=100$ is the number of trials [24].

(ii) Different sensing to stepping radius ratios: We repeated the simulations for the cases of the ratio of sensing to stepping ratio $\kappa=7$ and $\kappa=10$. We present the variation of probability of success in the Gaussian noise model in Figure 10.

(iii) Faster evader: We repeated the simulations for the case of faster evader. Assuming no noise, we present the variation of the probability of success in the top part of Figure 11. We observe that when the evader is at least $3 / 2$ times that of the pursuer, the proposed pursuer strategy is not efficient.

\section{B. Multiple pursuer problem}

Under the considered noise models:

- The SwEEP strategy remains unchanged. It terminates when an evader measurement is available. 

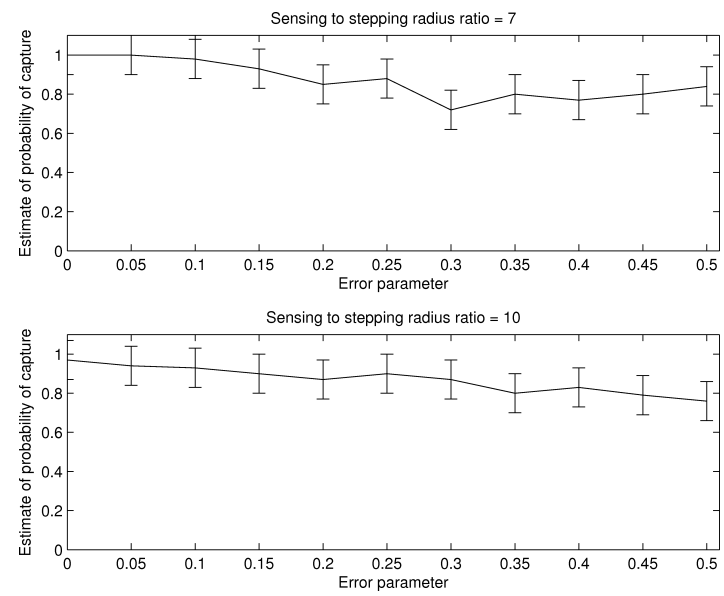

Fig. 10. Effect of varying the sensing to stepping radius ratio $\kappa$ in the single pursuer problem. For a particular evader strategy, we study how the capture probability varies for $\kappa=7$ and $\kappa=10$ with the noise parameter $\epsilon$, under Gaussian noise model. The error bars are in accordance with Figure 9.
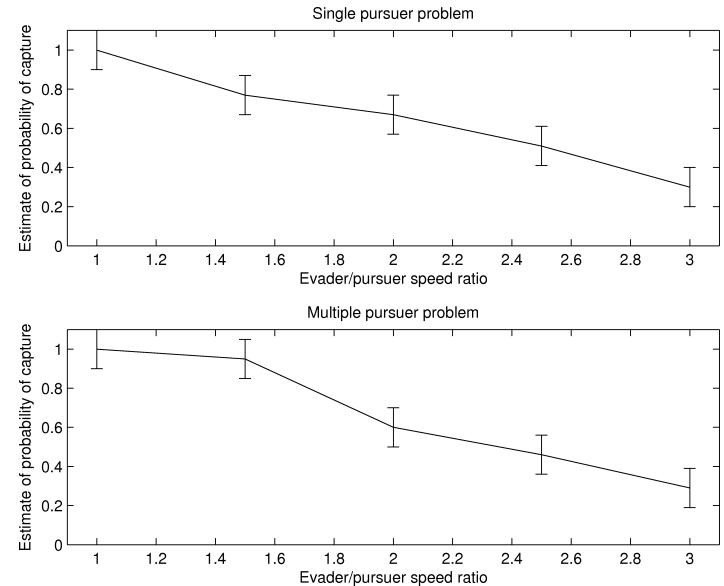

Fig. 11. Performance of Sweep-Pursuit-Capture strategy against a faster evader. For a particular evader strategy, we study how the capture probability varies for higher evader speeds, assuming no measurement noise. The top figure presents the single pursuer case and the bottom figure presents the multiple pursuer case. The error bars are in accordance with Figure 9.

- For Circumcenter and Planes strategies, the pursuers use the average of the available evader measurements to compute their next positions.

For the sake of simulations, we assume $N=7$ pursuers with $\kappa=5$ units and $r_{\text {step }}=1$ unit. We assume a square field of edge length 100 units, where the evader is initially placed at a uniformly randomly selected location. Upon detection, we assume that the evader moves away from the closest pursuer with some randomization. Specifically, it moves to a point on its motion circle, selected uniformly randomly in a sector of angle equal to 0.2 radians. This sector has its vertex at $e[t]$ and angle bisector parallel to the line $e\left[t_{\text {sweep }}\right] O$, where $t_{\text {sweep }}$ is the time when the evader is detected and $O$ is the circumcenter of the triangle $p_{2}\left[t_{\text {sweep }}\right], p_{6}\left[t_{\text {sweep }}\right]$ and $e\left[t_{\text {sweep }}\right]$. We study how the average capture time after detection varies with $\epsilon$. An upper limit of 1000 time steps was set to decide whether the strategy terminated in a failure.

The following is a summary of our findings: (i) Performance of the strategy with noisy measurements: The plots of probability of success of the strategy and average capture times after detection (given that the strategy terminates with capture) for both noise models versus the respective error parameters $\epsilon$ are shown in Figure 12. We observe a similar trend in the performance of the strategy in both noise models.
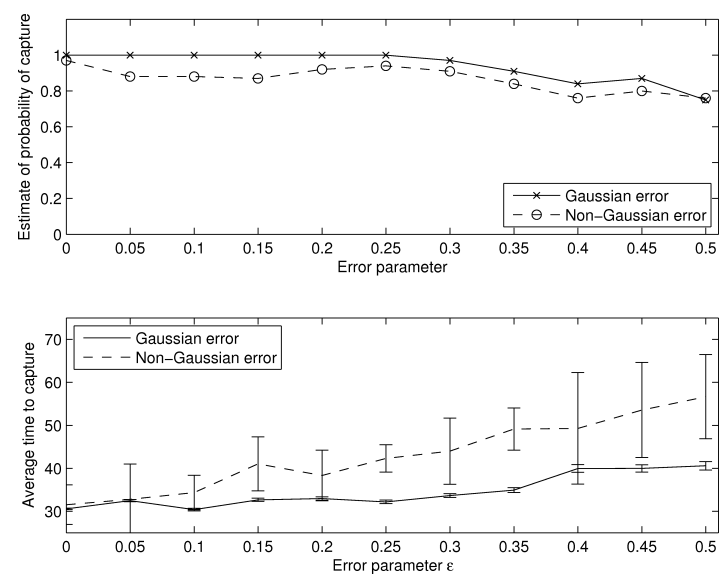

Fig. 12. Effect of measurement noise in the multiple pursuer problem. For a particular evader strategy, we study how the capture probability (top figure) and average capture time (bottom figure) given that the strategy succeeds, vary with the noise parameter $\epsilon$, under Gaussian and Non-gaussian error models. The error bars are in accordance with Figure 9.

(ii) Different number of pursuers: We repeated the simulations for the cases of the number of pursuers $N=10$ and $N=15$. We present the variation of probability of success in the Gaussian noise model in Figure 13.
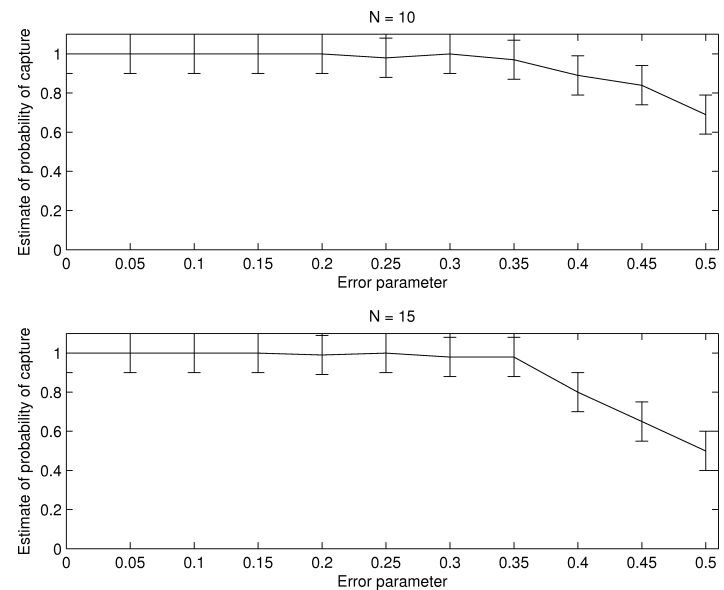

Fig. 13. Effect of varying the number of pursuers $N$ in the multiple pursuer problem. For a particular evader strategy, we study how the capture probability varies for $N=10$ and $N=15$ with the noise parameter $\epsilon$, under Gaussian noise model. The error bars are in accordance with Figure 9.

(iii) Faster evader: We repeated the simulations for the case of faster evader. Assuming no noise, we present the variation of the probability of success in the bottom part of Figure 11 . We observe that when the evader is at least 1.8 times that of the pursuers, the proposed pursuer strategy is not efficient. 


\section{BIOLOGICAL INTERPRETATIONS}

Our analysis in the previous sections can shed light on the trade-offs that predators face when deciding upon the group size. Based on our results from Section III-B, we now study how the group size of the pursuers varies with the evader availability in the multiple pursuer problem.

For simplicity, we assume a square field where the evader is initially located and denote by $\delta:=\frac{1}{\operatorname{diam}^{2}(\mathcal{G})}$ the evader density. From the results in Section III-B, an upper bound on the total time taken by the pursuers in all three phases of the strategy is given by

$$
\frac{1}{\delta(a N+b)}+\frac{c N}{\sqrt{\delta}(a N+b)}+k N,
$$

where $a:=2 r_{\text {step }}^{2} \sqrt{4 \kappa^{2}-25} / \pi, b:=\left(2 \pi \kappa-6 \sqrt{4 \kappa^{2}-25}\right) / \pi$, $c:=2 r_{\text {step }} \sqrt{4 \kappa^{2}-25}$ and $k:=\sqrt{4 \kappa^{2}-25}(1+1 / \sin \phi)+$ $18 \kappa \sqrt{4 \kappa^{2}-25}$ are constants independent of the number of predators $N$ or the evader density $\delta$.

From part (i) of Theorem III.7, we observe that when all other variables are kept constant, the lower bound on successful detection probability of the SWEEP strategy increases with $N$. However, a higher $N$ results into a greater time to capture the evader. This suggests a trade-off on the group size $N$ which we analyze as follows.

Let $\nu$ denote the nutritional content of the evader. We quantify the energy spent by each pursuer as the time taken to capture the evader. The energy gain from the pursuit is quantified as the amount of nutrition each participating pursuer receives from the evader. For a self-sustaining pursuit, we must have that the energy gained by each pursuer is at least equal to the energy spent during the hunt. Thus,

$$
\frac{\nu}{N} \geq \frac{1}{\delta(a N+b)}+\frac{c N}{\sqrt{\delta}(a N+b)}+k N .
$$

From this relation, we observe that for a given value of $\delta$, there exists an upper limit on the number of pursuers in the group for which it is advantageous for the pursuers to engage in a pursuit with the prospect of gaining energy. A plot of the upper limit on the group size $N$ versus the evader density $\delta$ is shown in Figure 14.

This analysis shows that for higher values of $\delta$, a larger number of pursuers can be accommodated in the trapping chain. This is consistent with observations in the biology literature by Caraco and Wolf [25] that have reported higher group size in foraging lions during the wet season (prey abundance), than in the dry season, (prey scarcity). Further, from our analysis, it also follows that for a given evader density, the higher the prey nutrition value $\nu$, the higher is the upper limit on the number of pursuers in the trapping chain. This is consistent with the observations reported by Griffiths [26] regarding how the size of hunting packs relate to the size of the prey relative to that of the predators.

\section{CONClusions And Future Directions}

We have addressed discrete-time pursuit-evasion problems in the plane with sensing capabilities restricted to a finite disc.

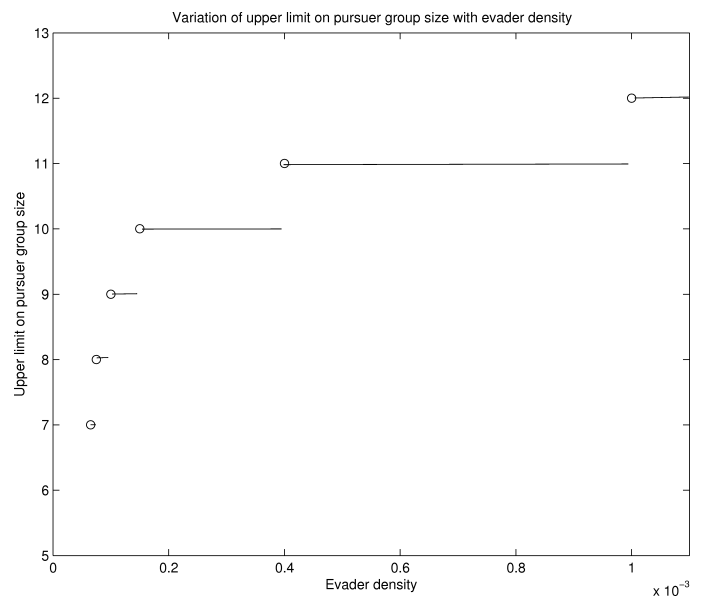

Fig. 14. Plot of maximum group size of pursuers that can be sustained versus the evader density $\delta$, for $\kappa=5, r_{\text {step }}=1, \nu=10000$.

We considered two variants of the pursuit-evasion in discretetime. The first involved a single pursuer and an evader in a bounded convex environment. The second was a formation design problem for multiple communicating pursuers to capture a single evader in a boundaryless environment. In both problems, the evader was initially located inside a bounded subset of the environment and moved only when detected. We proposed a Sweep-Pursuit-Capture strategy for both problems.

In the first problem, we gave sufficient conditions on the range of values taken by the ratio of sensing to stepping radius of the players so that the GREEDY pursuer strategy of moving towards the last-sensed evader position leads to the evader being trapped within the pursuer's sensing disc and finally to capture. We also gave conditions under which there exist locations from which the evader can escape. In the second problem, we have shown that the pursuers capture the evader with a certain probability that is independent of the initial evader location in a bounded region. We gave novel upper bounds on the total time taken to capture for both problems. We also presented simulation studies that addressed the performance of the strategies under sensing errors, different ratios of sensing to stepping radius, greater evader speed and different number of pursuers. Finally, on the basis of the obtained upper bound on the total capture time, we provided an upper bound on the group size of the pursuers for which the pursuit is advantageous from the point of view of gaining energy. The conclusions based on our analysis are consistent with observations reported in ecology literature.

In the future, it would be interesting to design efficient strategies for the single pursuer problem in complex environments. Also, considerations such as communication losses or errors in the multiple pursuer problem and more sophisticated sensing models for the players are potential future directions.

\section{ACKNOWLEDGMENTS}

This material is based upon work supported in part by ARO MURI Award W911NF-05-1-0219, ONR Award N00014-071-0721 and by the Institute for Collaborative Biotechnologies through the grant DAAD19-03-D-0004 from the U.S. Army 
Research Office. The authors would like to thank Prof. David Skelly for insightful conversations into animal behavior.

\section{REFERENCES}

[1] Y. Ho, A. E. Bryson, and S. Baron, "Differential games and optimal pursuit-evasion strategies," IEEE Transactions on Automatic Control, vol. 10, no. 4, pp. 385-389, 1965.

[2] H. Yamaguchi, "A cooperative hunting behavior by mobile-robot troops," International Journal of Robotics Research, vol. 18, no. 9, pp. 931-940, 1999.

[3] M. Pachter, "Simple motion pursuit-evasion differential games," in Mediterranean Conference on Control and Automation, Lisbon, Portugal, Jul. 2002, Electronic Proceedings.

[4] J. Sgall, "A solution of David Gale's lion and man problem," Theoretical Computational Science, vol. 259, no. (1-2), pp. 663-670, 2001

[5] S. Kopparty and C. V. Ravishankar, "A framework for pursuit evasion games in $\mathbb{R}^{n}$," Information Processing Letters, vol. 96, no. 3, pp. 114122, 2005.

[6] L. Alonso, A. S. Goldstein, and E. M. Reingold, "Lion and Man: Upper and lower bounds," ORSA Journal of Computing, vol. 4, no. 4, pp. 447452, 1992.

[7] S. Alexander, R. Bishop, and R. Ghrist, "Pursuit and evasion in nonconvex domains of arbitrary dimension," in Robotics: Science and Systems II (Philadelphia PA), G. S. Sukhatme, S. Schaal, W. Burgard, and D. Fox, Eds. Cambridge, MA: MIT Press, 2007.

[8] S. M. LaValle and J. Hinrichsen, "Visibility-based pursuit-evasion: The case of curved environments," IEEE Transactions on Robotics and Automation, vol. 17, no. 2, pp. 196-201, 2001.

[9] L. J. Guibas, J. C. Latombe, S. M. LaValle, D. Lin, and R. Motwani, "A visibility-based pursuit-evasion problem," International Journal of Computational Geometry \& Applications, vol. 9, no. 4-5, pp. 471-493, 1999.

[10] V. Isler, S. Kannan, and S. Khanna, "Randomized pursuit-evasion in a polygonal environment," IEEE Transactions on Robotics, vol. 5, no. 21, pp. 875-884, 2005.

[11] B. P. Gerkey, S. Thrun, and G. Gordon, "Visibility-based pursuit-evasion with limited field of view," International Journal of Robotics Research, vol. 25 , no. 4, pp. 299-315, 2006.

[12] I. Shevchenko, "Successive pursuit with a bounded detection domain," Journal of Optimization Theory \& Applications, vol. 95, no. 1, pp. 25-48, oct 1997.

[13] Z. Cao, M. Tan, L. Li, N. Gu, and S. Wang, "Cooperative hunting by distributed mobile robots based on local interaction," IEEE Transactions on Robotics, vol. 22, no. 2, pp. 403-407, 2006.

[14] V. Isler, S. Kannan, and S. Khanna, "Randomized pursuit-evasion with local visibility," SIAM Journal on Discrete Mathematics, vol. 1, no. 20, pp. 26-41, 2006.

[15] J. P. Hespanha, H. J. Kim, and S. S. Sastry, "Multipleagent probabilistic pursuit-evasion games," Electrical Engineering and Computer Science, University of California at Berkeley, Tech. Rep., Aug. 1999, available electronically at http://www.ece.ucsb.edu/ hespanha/published.

[16] R. Vidal, O. Shakernia, H. Kim, D. H. Shim, and S. Sastry, "Probabilistic pursuit-evasion games: Theory, implementation and experimental evaluation," IEEE Transactions on Robotics and Automation, vol. 18, no. 2, pp. 662-669, 2002.

[17] Y. Gabriely and E. Rimon, "Spanning-tree based coverage of continuous areas by a mobile robot," Annals of Mathematics and Artificial Intelligence, vol. 31, no. 1-4, pp. 77-98, 2001.

[18] S. D. Bopardikar, F. Bullo, and J. P. Hespanha, "Sensing limitations in the Lion and Man problem," in American Control Conference, New York, Jul. 2007, pp. 5958-5963.

[19] _ - "Cooperative pursuit with sensing limitations," in American Control Conference, New York, Jul. 2007, pp. 5394-5399.

[20] C. Packer and L. Ruttan, "The evolution of cooperative hunting," The American Naturalist, vol. 132, no. 2, pp. 159-198, 1988.

[21] S. K. Gazda, R. C. Connor, R. K. Edgar, and F. Cox, "A division of labour with role specialization in group-hunting bottlenose dolphins (Tursiops truncatus) off Cedar Key, Florida," Proceedings of the Royal Society B: Biological Sciences, vol. 272, no. 1559, pp. 135-140, 2005.

[22] B. L. Partridge and T. J. Pitcher, "The sensory basis of fish schools: Relative roles of lateral line and vision," Journal of Comparative Physiology A, vol. 135 , no. 4 , pp. $315-325,1980$.
[23] S. D. Bopardikar, F. Bullo, and J. P. Hespanha, "On discrete-time pursuit-evasion games with sensing limitations," Center for Control, Dynamical Systems and Computation. University of California at Santa Barbara, Tech. Rep. CCDC-08-0313, 2008, available electronically at http://ccdc. mee. ucsb.edu.

[24] R. E. Walpole, R. H. Myers, and S. L. Myers, Probability and Statistics for Engineers and Scientists, 6th ed. New Jersey, USA: Prentice Hall, 1998.

[25] T. Caraco and L. L. Wolf, "Ecological determinants of group sizes of foraging lions," The American Naturalist, vol. 109, no. 967, pp. 343-352, May-June 1975.

[26] D. Griffiths, "Foraging costs and relative prey size," The American Naturalist, vol. 116, no. 5, pp. 743-752, 1980.

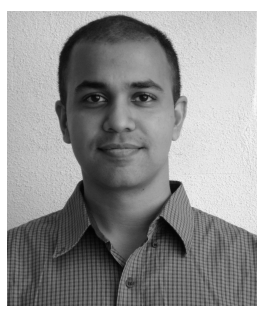

Shaunak D. Bopardikar received the Bachelor of Technology (B.Tech.) and Master of Technology (M.Tech.) degrees in Mechanical Engineering from Indian Institute of Technology, Bombay, India, in 2004. He is currently working toward the Ph.D. degree in Mechanical Engineering at the University of California at Santa Barbara, USA.

His research interests include pursuit-evasion games, motion coordination and cooperative control.

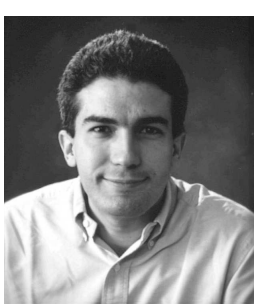

Francesco Bullo received the Laurea degree in Electrical Engineering from the University of Padova, Italy, in 1994, and the Ph.D. degree in Control and Dynamical Systems from the California Institute of Technology in 1999. From 1998 to 2004, he was an Assistant Professor with the Coordinated Science Laboratory, University of Illinois at UrbanaChampaign. He is currently a Professor in the Mechanical Engineering Department, University of California, Santa Barbara. His research interests include motion planning and coordination for autonomous vehicles, motion coordination for multi-agent networks, and geometric control of mechanical systems. He is the coauthor (with Andrew D. Lewis) of the "Geometric Control of Mechanical Systems" York: Springer, 2004, 0387-22195-6). He is currently serving on the editorial board of the IEEE Transactions on Automatic Control and of the SIAM Journal of Control and Optimization.

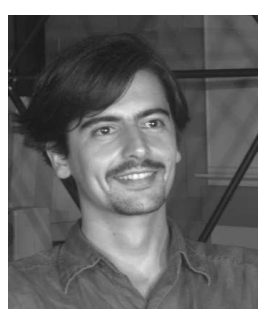

João P. Hespanha received his Ph.D. degree in electrical engineering and applied science from Yale University, New Haven, Connecticut in 1998. From 1999 to 2001, he was Assistant Professor at the University of Southern California, Los Angeles. He moved to the University of California, Santa Barbara in 2002, where he currently holds a Professor position with the Department of Electrical and Computer Engineering. Prof. Hespanha is Associate Director for the Center for Control, Dynamical-systems, and Computation (CCDC), Vice-Chair of the Department of Electrical and Computer Engineering, and a member of the Executive Committee for the Institute for Collaborative Biotechnologies (ICB). From 20042007 he was an associate editor for the IEEE Transactions on Automatic Control.

His current research interests include hybrid and switched systems; the modeling and control of communication networks; distributed control over communication networks (also known as networked control systems); the use of vision in feedback control; and stochastic modeling in biology.

Dr. Hespanha is the recipient of the Yale Universitys Henry Prentiss Becton Graduate Prize for exceptional achievement in research in Engineering and Applied Science, a National Science Foundation CAREER Award, the 2005 best paper award at the 2nd Int. Conf. on Intelligent Sensing and Information Processing, the 2005 Automatica Theory/Methodology best paper prize, and the 2006 George S. Axelby Outstanding Paper Award. Dr. Hespanha is a Fellow of the IEEE and an IEEE distinguished lecturer since 2007. 